\title{
Niveles de estrés y síndrome de burnout en urólogos mexicanos. ¿Cómo nos encontramos?
}

Diego Antonio Preciado-Estrella, Javier A. Herrera-Muñoz, José Gómez-Sánchez, Pascual Cortés-Raygoza, Mario Ortega-González, Iván Calvo-Vázquez y colaboradores.

\section{Resumen}

ANTECEDENTES: Según la Red del Trabajo e Información Ocupacional de Estados Unidos, la urología fue la especialidad con mayores niveles de estrés en 2016, estimada entre 900 diferentes profesiones, por encima de los policías, bomberos o anestesiólogos.

OBJETIVOS: Determinar la prevalencia de estrés y síndrome de burnout en urólogos mexicanos e identificar las causas implicadas con su origen.

MATERIALES Y MÉTODOS: Estudio descriptivo, transversal y analítico, efectuado a través de la versión abreviada del cuestionario de estrés y burnout en urólogos mexicanos. Se registraron características sociodemográficas. Para el análisis estadístico se utilizaron variables descriptivas (tendencia central, desviación estándar), con la finalidad de conocer el perfil demográfico. Cada individuo se clasificó según su nivel de estrés en: bajo, intermedio o alto, cuyos datos se analizaron mediante estadística inferencial (tablas de contingencia, $\chi^{2}$ y prueba exacta de Fisher). Los datos recabados se analizaron con el programa SPSS ${ }^{\circledR}$ IMB-versión-21. Se consideró estadísticamente significativo el valor de $\mathrm{p}<0.05$.

RESULTADOS: Se aplicaron 137 encuestas al azar. La media de edad fue 45 años (DE \pm 9), 59\% de los urólogos ejercía la práctica mixta (institución pública y privada) y la cirugía fue la actividad profesional más estresante (31\%). Se registraron 75 (54\%) urólogos con niveles bajos de estrés, $41 \%$ con moderado y $2.1 \%$ con severos. Los síntomas más frecuentes fueron astenia $(17.5 \%)$, atracones de comida $(17.4 \%)$ y cefalea (12\%). Las variables implicadas con el estrés de los urólogos mexicanos fueron: edad joven, trabajar en instituciones públicas y obtener ingresos económicos menores de 50,000 pesos mensuales $(p=0.05)$.

CONCLUSIONES: El estrés es una alteración habitual en la urología; sin embargo, los urólogos mexicanos no muestran niveles tan altos comparados con los de otros países. La edad joven, sobrecarga laboral en instituciones públicas y los bajos ingresos monetarios son factores implicados con el estrés.

PALABRAS CLAVE: Estrés; burnout, urólogo.
División de Urología, Hospital General Dr. Manuel Gea González, Ciudad de México.

Recibido: octubre 2017

Aceptado: marzo 2018

Correspondencia

Diego Antonio Preciado Estrella

dr.diegopreciado@gmail.com

Este artículo debe citarse como

Preciado-Estrella DA, Herrera-Muñoz JA, GómezSánchez J, Cortés-Raygoza P, Ortega-González M, Calvo-Vázquez I y col. Niveles de estrés y síndrome de burnout en urólogos mexicanos. ¿Cómo nos encontramos? Rev Mex Urol. 2018 marzo-abril;78(2):98-104. DOI: https://doi.org/10.24245/revmexurol.v78i2.1741 
Preciado-Estrella DA y col. Niveles de estrés y burnout en urólogos

Rev Mex Urol. 2018 March-April;78(2):98-104.

\title{
Levels of stress and burnout in Mexican urologists. How are we doing?
}

\begin{abstract}
Diego Antonio Preciado-Estrella, Javier A. Herrera-Muñoz, José Gómez-Sánchez, Pascual Cortes-Raygoza, Mario Ortega-González, Iván Calvo-Vázquez y colaboradores.
\end{abstract}

\begin{abstract}
BACKGROUND: According to the U.S. Occupational Information Network, out of 900 professions, that of urologist was one of the most stressful in 2016, more so than that of police officer, fire fighter, or anesthesiologist.
\end{abstract}

OBJECTIVES: To determine the prevalence of stress and burnout in Mexican urologists and identify their causes.

MATERIALS AND METHODS: A descriptive, analytic, cross-sectional study was conducted, applying a short version of the stress and burnout inventory to Mexican urologists. The sociodemographic characteristics were registered. Descriptive statistics (central tendency, standard deviation) were used to describe the demographic profile. Each individual was classified by stress level: mild, intermediate, or high, employing inferential statistics (contingency tables, $\chi^{2}$ test, and the Fisher's exact test). The data were analyzed using the SPSS ${ }^{\circledR}$ IMB-Version-21 program. Statistical significance was set at a $\mathrm{p}<0.5$.

RESULTS: One hundred thirty-seven questionnaires were randomly applied. Mean age of the urologists was 45 years $(S D \pm 9), 59 \%$ worked at both a public healthcare institution and in private practice (mixed practice), and surgery was the most stressful professional activity (31\%). Stress levels were mild in 75 urologists (54\%), moderate in $41 \%$, and severe in $2.1 \%$. The most frequent symptoms were asthenia $(17.5 \%)$, binge eating (17.4\%), and headache (12\%). The variables that had an impact on stress in the Mexican urologist were: young age, working in a public health institution, and a monthly income under 50,000.00 MXN ( $p=0.05)$.

CONCLUSIONS: Stress is a common condition in urologic practice, but the levels of stress in the urologists surveyed were not as high as those reported in other countries. Youth, work overload, especially in the public healthcare institutions, and low income were the factors involved.

KEYWORDS: Stress; Burnout; Urologist.

\section{ANTECEDENTES}

El estrés es una respuesta natural sin la que los seres humanos no habrían supervivido a lo largo de la evolución. Por tratarse de una condición desagradable es temida y evitada por todos. Los fenómenos biológicos originados en el cuerpo luego de una situación estresante (por ejemplo accidentes) implican la liberación de cortisol y catecolaminas, pero cuando la situación se vuelve crónica, esas hormonas persisten elevadas y provocan cambios en el organismo y la 
personalidad. La respuesta o reacción al estrés es diferente entre los individuos, incluso las situaciones que para alguien pueden ser amenazantes, para otros no lo son.

El término burnout o síndrome del quemado fue empleado por primera vez en 1974 por Fredenberger ${ }^{1} y$ hace referencia al estado físico y mental subóptimo, que resulta del estrés laboral crónico al que se somete un individuo y repercute en su nivel de efectividad, salud física y mental. La triada clásica del burnout consiste en: 1) agotamiento emocional, 2) despersonalización, entendida como actitud negativa-hostil y 3 ) deficiente sentido de realización personal. ${ }^{2}$

El burnout puede ocurrir en cualquier ámbito profesional. La práctica médica es una de las profesiones más afectadas y el estrés se manifiesta como cansancio crónico, despersonalización (incluso puede provocar un trato déspota hacia los pacientes y compañeros de trabajo) y falta de logros personales y profesionales que conducen a un estado de distimia y adinamia crónica, en el que finalmente resulta en un deficiente desempeño, falta de entusiasmo, compromiso y errores continuos. ${ }^{3}$ Un estudio efectuado en la Clínica Mayo señaló $54 \%$ de burnout en los médicos, comparado con $28 \%$ de la población general, incluso la urología fue una de las especialidades más afectadas, con niveles incluso de $64 \%{ }^{4}$

Desde la década de 1980 Maslach $^{5}$ estudió los efectos biopsicosociales que el estrés crónico causa al organismo y describió un cuestionario ampliamente difundido para evaluar el burnout. Este instrumento analiza 22 aspectos físicos y psicológicos, entre los que destacan: pérdida de confianza, entusiasmo, responsabilidad, apatía laboral, actitud negativa, irritabilidad y agresividad, cansancio y falta de interés que resultan en depresión.

Puesto que el estrés desmedido y mal canalizado es en la actualidad un factor de riesgo para la salud y las relaciones sociales, entenderlo resulta fundamental para tratarlo.

El objetivo de este estudio es determinar la prevalencia de estrés en urólogos mexicanos e identificar las causas implicadas con su origen.

\section{MATERIALES Y MÉTODOS}

Estudio descriptivo, transversal y analítico, efectuado a través de la versión abreviada del cuestionario de Maslach, modificado por GarcíaIzquierdo, que evalúa los síntomas referidos en el Cuadro 1. ${ }^{6}$ en urólogos mexicanos. De acuerdo con el cuestionario, los síntomas relacionados con el estrés y burnout se clasifican según su manifestación en: nunca ( 1 punto), casi nunca (2 puntos), pocas veces (3 puntos), algunas veces (4 puntos), con relativa frecuencia (5 puntos) y con mucha frecuencia (6 puntos).

También se analizaron factores socioeconómicos, familiares y laborales en cada urólogo, con la finalidad de asociarlos con diferentes rubros relativos al estrés. ${ }^{7}$ Se les pidió a los participantes responder de forma anónima el cuestionario.

A cada respuesta se le asignó un puntaje, del 1 al 6, en función de la frecuencia de los sínto-

Cuadro 1. Síntomas relacionados con el estrés y burnout (versión modicada de Garcia-Izquierdo ${ }^{6}$ )

- Imposibilidad de conciliar el sueño

- Cefalea

- Molestias gastrointestinales

- Agotamiento

- Tendencia a comer, beber o fumar más de lo habitual

- Disminución del interés sexual

- Respiración entrecortada o sensación de ahogo

- Disminución del apetito

- Temblores musculares (tics)

- Piquetes o sensaciones dolorosas en el cuerpo

- Tentaciones fuertes de no levantarse

- Sudoración o palpitaciones 
mas de cada individuo y la suma de todas las respuestas permitió clasificar la severidad del estrés en: leve (menos de 25), moderado (25 a 48) y severo (más de 49).

Las encuestas fueron aplicadas al azar, durante el congreso de la Sociedad Mexicana de Urología, por médicos residentes del Hospital General Dr. Manuel Gea González a urólogos graduados de distintas edades, siempre guardando el respeto y la confidencialidad de las respuestas y con autorización de la Sociedad Mexicana de Urología Colegio de Profesionistas. La información recabada se integró en una base de datos y a partir de ella se desarrollaron los análisis y pruebas estadísticas correspondientes (tablas de contingencia, $\chi^{2}$ y prueba exacta de Fisher) en el programa de cómputo SPSS versión 21 (IBM Corp, 2013). Se consideró estadísticamente significativo el valor de $p<0.5$.

\section{RESULTADOS}

Se aplicaron 137 encuestas a urólogos mexicanos. El grupo etario más común fue el de 30 a 50 años (promedio $45 \pm 9$ años; $56.2 \%$ ), seguido del grupo de 51 a 65 años (33\%). El 67\% era casado, $22 \%$ soltero y $13 \%$ divorciado. En cuanto al tipo de práctica profesional, 59\% de los urólogos laboraba en la práctica mixta (institución pública y privada), $22 \%$ en la privada y $19 \%$ en la pública.

Al cuestionar qué actividad de su práctica profesional resultaba más estresante, la cirugía abierta fue señalada por $31 \%$ y la consulta por $22 \%$. El ingreso económico promedio mensual más frecuente fue de $\$ 50,000$ a 150,000 pesos mexicanos (40\%), seguido de menos de $\$ 50,000$ (34\%) y mayor de \$150,000 (25\%).

Con base en las respuestas de cada individuo, la prevalencia y severidad del estrés fue baja en 75 (54\%) urólogos, moderada en 58 (41\%) y severa en $3(2.1 \%)$ (Figura 1).

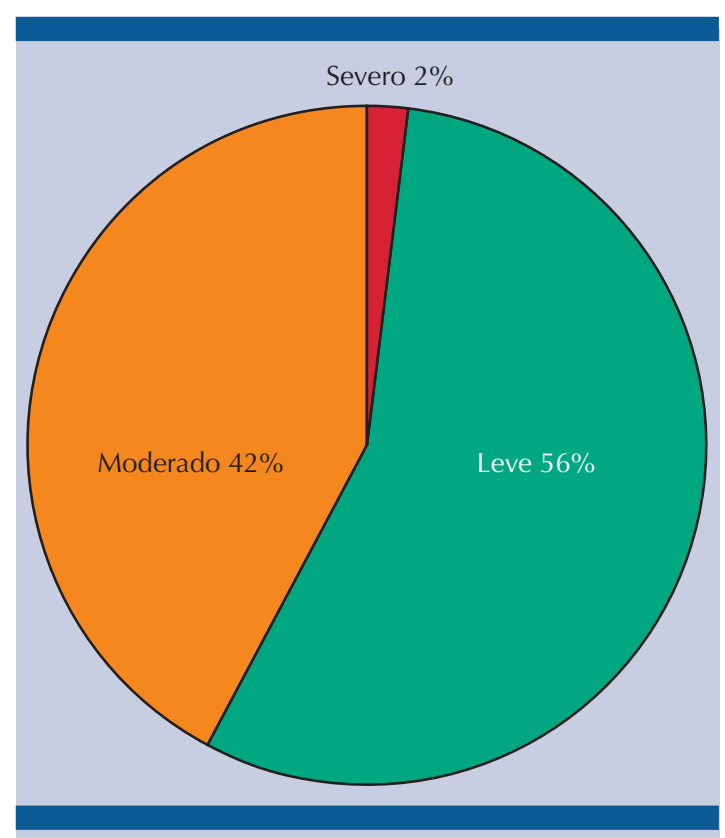

Figura 1. Prevalencia de niveles de estrés en urólogos mexicanos.

En la revisión sistemática de cada respuesta del cuestionario de estrés se encontró que el síntoma más frecuente fue el agotamiento (17.5\%), seguido de los atracones de comida y bebida $(17.4 \%)$ y la cefalea $(12 \%)$.

Se realizó el análisis bivariado múltiple, mediante pruebas de estadística inferencial (tablas de contingencia, $\chi^{2}$ y prueba exacta de Fisher), para establecer las asociaciones de interés entre las respuestas obtenidas (Cuadro 2). Al comparar los niveles de estrés, según la edad mediante la prueba $\chi^{2}$, los urólogos más jóvenes obtuvieron un promedio más alto (25.1 puntos) que los de mayor edad (21.2 puntos), por lo que ser urólogo joven se asoció con niveles altos de estrés $\left(\chi^{2}=16.24, \mathrm{GL}=3, p=0.01\right)$. Sin embargo, cuando se consideró la experiencia profesional en años, no se encontraron diferencias estadísticamente significativas, aunque los urólogos más adiestrados obtuvieron puntuaciones más bajas $(p=0.21$ ) 
Cuadro 2. Niveles de estrés por grupos, según el puntaje del cuestionario aplicado. ${ }^{6}$

\begin{tabular}{|c|c|c|c|}
\hline \multirow[t]{2}{*}{ Factor } & \multirow[t]{2}{*}{ Categoría } & \multicolumn{2}{|c|}{$\begin{array}{c}\text { Análisis bivariado } \\
\text { múltiple }\end{array}$} \\
\hline & & $\begin{array}{l}\text { Puntaje del } \\
\text { cuestionario }\end{array}$ & $\mathbf{p}$ \\
\hline Edad & $\begin{array}{l}<45 \text { años } \\
>45 \text { años }\end{array}$ & $\begin{array}{l}25.13 \\
21.25\end{array}$ & $\begin{array}{c}0.01 \\
\text { (tablas de } \\
\text { contingen- } \\
\text { cia) }\end{array}$ \\
\hline $\begin{array}{l}\text { Años de } \\
\text { práctica } \\
\text { como } \\
\text { especialista } \\
\text { en urología }\end{array}$ & $\begin{array}{l}<5 \text { años } \\
5 \text { a } 10 \text { años } \\
>10 \text { años }\end{array}$ & $\begin{array}{c}25.9 \\
24.39 \\
22.5\end{array}$ & $\begin{array}{c}0.21 \\
\left(\chi^{2}\right)\end{array}$ \\
\hline $\begin{array}{l}\text { Tipo de } \\
\text { práctica } \\
\text { (institución) }\end{array}$ & $\begin{array}{l}\text { Mixta } \\
\text { Privada } \\
\text { Pública }\end{array}$ & $\begin{array}{c}25.15 \\
22.6 \\
24.6\end{array}$ & $\begin{array}{c}<0.05 \\
\left(\chi^{2}\right)\end{array}$ \\
\hline Estado civil & $\begin{array}{c}\text { Soltero } \\
\text { Casado } \\
\text { Divorciado }\end{array}$ & $\begin{array}{c}27.7 \\
22.95 \\
19.75\end{array}$ & $\begin{array}{c}0.75 \\
\left(\chi^{2}\right)\end{array}$ \\
\hline Ingresos & $\begin{array}{c}<50,000 \mathrm{MN} \\
50-150,000 \mathrm{MN} \\
>150,000 \mathrm{MN}\end{array}$ & $\begin{array}{l}25.5 \\
24.7 \\
22.6\end{array}$ & $\begin{array}{c}<0.05 \\
\text { (prueba } \\
\text { de Fisher) }\end{array}$ \\
\hline
\end{tabular}

MN: moneda nacional.

En cuanto al tipo de práctica institucional y el nivel de estrés, los urólogos que laboran en la práctica mixta tuvieron mayor puntuación (18\%) que quienes trabajan en instituciones públicas $(12 \%)$ o privadas $(3 \%)(p<0.05)$.

En lo que a estado civil respecta, los solteros obtuvieron puntuaciones de estrés más altas (39\%) que los casados (14\%). Los niveles más bajos de estrés se observaron en los urólogos divorciados; sin embargo, no hubo diferencias estadísticamente significativas $\left(\chi^{2}=9.03, G L 7, p=0.75\right)$.

Respecto del ingreso económico, el grupo con los niveles más altos de estrés fue el de los urólogos que perciben menos ingresos (19\%) con síntomas de burnout, seguido del grupo con ingresos intermedios (15\%). En el grupo con ingresos altos sólo 1/34 (3\%) urólogos refirió estrés considerable $(p=0.05)$.

\section{DISCUSIÓN}

Desde la década pasada Böhle ${ }^{8}$ y sus colaboradores evidenciaron la prevalencia de estrés y síndrome de burnout en urólogos alemanes mediante la escala de Maslach. En ese estudio se enfocaron en aspectos como la edad y el desempeño profesional y los compararon con especialistas de una institución pública versus privada. Entre sus resultados encontraron que los urólogos con amplia experiencia, mayores de 45 años de edad y que laboran en una institución privada tuvieron menores niveles de estrés, lo que coincide con los resultados obtenidos en este estudio.

Hace poco la Asociación Americana de Urología reportó los resultados del censo 2016, en el que incluyó el cuestionario de Maslach, y cuyo análisis fue realizado por North y su grupo, ${ }^{9}$ quienes concluyeron que $38.8 \%$ de los urólogos cumplieron con los criterios del síndrome de burnout, de los que $17.2 \%$ obtuvieron una calificación alta de agotamiento emocional despersonalización. Los factores de riesgo identificados para padecer estrés en los urólogos norteamericanos incluyeron: mayor número de pacientes atendidos por semana, edad joven y dedicarse a áreas de la urología diferentes a la pediatría u oncología, mientras que trabajar solo en un centro académico resultó ser un factor protector. Los resultados del censo norteamericano fueron similares en algunos aspectos a los nuestros; sin embargo, no son equiparables debido a las diferencias en los sistemas de salud y las variables medidas.

Por lo que se refiere al nivel de estrés en urólogos en formación, un estudio francés publicado por Roumiguié, ${ }^{10}$ quien también aplicó el cuestionario de Maslach, demostró una prevalencia de 
síndrome de burnout severo de 24\%, en donde destacaron los síntomas de despersonalización, mientras que tener una pareja o un pasatiempo resultaron factores protectores. Evaluar la prevalencia de estrés entre los urólogos en formación en México implica considerar diferentes variables a las empleadas en este estudio, que sin duda será un área interesante por estudiar a futuro.

El Reino Unido reporta niveles de agotamiento emocional y despersonalización de 28.6 y $29.9 \%$, respectivamente. ${ }^{11}$ En esta población los principales factores de riesgo identificados para padecer estrés fueron: sobrecarga laboral y administrativa, falta de recursos institucionales, edad joven y temas relacionados con pensiones y retiro profesional.

El estrés laboral y síndrome de burnout son trastornos con elevada prevalencia en los médicos más que en otros profesionistas. Se ha estudiado la prevalencia de estrés en diversas especialidades médicas y quirúrgicas; sin embargo, en los urólogos es limitada esta información. EI estudio de North, ${ }^{12}$ efectuado en urólogos de Nueva York, señaló al sexo femenino y tener problemas personales o familiares como factores de riesgo implicados con el síndrome de burnout. En nuestro estudio, todos los médicos encuestados fueron hombres, quizá debido a la baja prevalencia de mujeres en la urología mexicana. Un estudio realizado en 2009 por el Colegio Americano de Cirujanos ${ }^{13}$ demostró que $32 \%$ de los cirujanos de Estados Unidos tuvieron agotamiento emocional, con altos niveles de despersonalización (26\%) y bajo sentido de realización personal (13\%), incluso 40\% cumplió con los criterios de síndrome de burnout.

De manera similar, la encuesta efectuada en 2016 por Jason ${ }^{14}$ demostró que la prevalencia de síndrome de burnout en los urólogos pediatras fue de $14 \%$, cifra menor a la de los urólogos que atienden pacientes adultos, considerándose incluso una de las más bajas entre todas las especialidades. En nuestro estudio no se evalúo por separado la práctica de adultos versus pediátrica.

De acuerdo con Sonia Lupien, existen 4 características para generar una situación estresante: 1) novedad, 2) elementos imprevistos, 3) sensación de descontrol y 4) amenaza para la personalidad. ${ }^{15}$ Mientras más de estos factores estén implicados en determinada situación (por ejemplo, una nefrectomía laparoscópica), mayor será el estrés del individuo; así pues, si la situación estresante se repite continuamente y no se desarrollan elementos protectores (mayor experiencia, control de la situación), el umbral al estrés disminuye y percibe al sujeto más reactivo, incluso hipersensible a situaciones menores, por ejemplo al efectuar una cistoscopia.

El desequilibrio entre la profesión y la vida personal se asocia con altos niveles de estrés y síndrome de burnout. Una encuesta aplicada a cirujanos, que evaluó conflictos personales y laborales, reveló que $47 \%$ tuvo alguna problemática familiar relacionada con la profesión. ${ }^{16}$ Lo anterior coincide parcialmente con nuestros resultados, donde los urólogos solteros suelen estar más estresados que los casados; por su parte, los divorciados tuvieron niveles más bajos de estrés.

\section{CONCLUSIONES}

La prevalencia de estrés y síndrome de burnout es cada vez más elevada en los urólogos de todo el mundo. Una de las causas que incrementan estos trastornos se relaciona con la tasa de envejecimiento de la población, por lo que la carga laboral en urología se incrementa de manera proporcional al aumentar la prevalencia de síntomas urinarios, disfunción eréctil, neoplasias e incontinencia. A pesar de lo anterior, en este estudio se encontraron diferencias (menores) en cuanto a los niveles de estrés de los urólogos mexicanos respecto a los del resto del mundo. 
Entre los factores identificados en los urólogos mexicanos con mayor estrés destacan la edad joven, los bajos ingresos económicos y la sobrecarga laboral. La mejor manera de enfrentarse al estrés es identificar sus cuatro características principales y establecer una estrategia efectiva para contrarrestarlas.

El síndrome de burnout es un problema grave en los trabajadores de la salud, que amenaza al número de médicos disponibles y la calidad en la atención. La disminución y administración racional de la carga laboral, en conjunto con la consolidación de una curva de aprendizaje y soporte laboral apropiados representan estrategias convenientes para contrarrestar el estrés en los urólogos mexicanos.

\section{Colaboradores}

Erick A. Hernández-Méndez, Gerardo Fernández-Noyola, Mauricio Cantellano-Orozco, Carlos Martínez-Arroyo, Gustavo MoralesMontor, Carlos Pacheco-Gahbler.

\section{Conflicto de interés}

El Dr. Carlos Martínez Arroyo colabora con la empresa BARD.

\section{REFERENCIAS}

1. Freudenberger JH. Staff burn-Out. J Soc Issues 1974;30(1):159-165.

2. Wright TA, Cropanzano R. Emotional exhaustion as a predictor of job performance and voluntary turnover. J Appl Psychol 1998;83(3):486-93.
3. Cull AM. Studying stress in care givers: art or science? Br J Cancer 1991;64(6):981-4.

4. Shanafelt T, Hasan O, Dyrbye LN, et al. Changes in burnout and satisfaction with work-life balance in physicians and the general U.S. working population between 2011 and 2014. Mayo Clin Proc 2015;90(12):1600-1613.

5. Maslach C, Jackson SE. Maslach Burnout Inventory Manual. $2^{\text {nd }}$ ed. Palo Alto: Consulting Psychologists Press, 1986.

6. García-Izquierdo M, Velandrino A. EPB: Una escala para la evaluación del burnout profesional de las organizaciones. Anales Psicol 1992;8(1-2):131-138.

7. McEwen BS, Gianaros PJ. Central role of the brain in stress and adaptation: links to socioeconomic status, health, and disease. Ann N Y Acad Sci 2010;1186:190-222.

8. Böhle A, Baumgärtel M, Götz ML, Müller EH, Jocham D. Burn-out of urologists in the county of Schleswig-Holstein, Germany: a comparison of hospital and private practice urologists. J Urol 2001;165(4):1158-2001.

9. North CA, McKenna HP, Fang R, et al. Burnout in urology: results from the AUA Census. J Urol 2017;197(4S).

10. Roumiguie $M$, Gamé X, Bernhard JC, Bigot $P$, et al. Les urologues en formation ont-ils un síndrome d'épuisement professionnel? Évaluation par le Maslach Burn-out Inventory (MBI). Progrès Urologie 2011;21:636-641.

11. O'Kelly, Manecksha RP, Quinlan DM, Reid A, et al. Rates of self-reported 'burnout' and causative factors amongst urologists in Ireland and the UK: a comparative crosssectional study. BJUI Int 2015;117(2):363-72.

12. North A. Review Article: Physician Burnout in urology. Urol Pract 2017;4(2):155-161.

13. Romani M, Ashkar K. Burnout among physicians. Libyan Journal of Medicine 2014;9: https://doi.org/10.3402/ljm. v9.23556.

14. Jason Au, Elizondo AR, Roth RD. Surgeon Burnout among American Pediatric Urologists. Urol Pract 2017;4(3):264268.

15. Lupien S. The history of the Science of Stress: From Hans Selye to the discovery of anti-inflammatory medication. Sante Ment Que 2015;40(2):275-86.

16. Troppmann KM, Palis BE, Goodnight JE, Ho HS, Troppmann C. Career and lifestyle satisfaction among surgeons: what really matters? The National Lifestyles in Surgery Today Survey. J Am Coll Surg 2009;209(2):160-169. 